Otimização do Processo de Extração de Compostos Fenólicos

Antioxidantes do Jiló (Solanum gilo Radi) e Aplicação na Estabilidade Oxidativa do Óleo de Soja

\author{
da Silva, C. F. G.; Suzuki, R. M.; Canesin, E. A.; Tonin, L. T. D.* \\ Rev. Virtual Quim., 2017, 9 (2), 729-739. Data de publicação na Web: 22 de fevereiro de 2017 \\ http://rvq.sbq.org.br

\section{Optimization of the Extraction Process of Phenolic Antioxidant Compounds of Jelly (Solanum gilo Radi) and Application in the Oxidative Stability of Soybean Oil}

\begin{abstract}
The objective of this study was evaluate the effects of jelly fruit extracts as a natural antioxidant in soybean oil subjected to an accelerated storage test and optimize the best extraction conditions of antioxidant compounds of jelly (Solanum gilo Radi) dehydrated at temperatures of 40,6080 and $100{ }^{\circ} \mathrm{C}$, and thus to evaluate the influence of temperature on antioxidant activity. The antioxidant activity was determined by the DPPH radical scavenging assay and total phenolic compounds using the Folin-Ciocalteu method. The conditions that provided the most jelly extract with antioxidant activity were dehydrated at $60^{\circ} \mathrm{C}$ using $5.0 \mathrm{~g}$ of flour, $60 \mathrm{~mL}$ of methanol/water $20 \%(\mathrm{v} / \mathrm{v})$ for 24 hours and protected from light. The monitoring of oil oxidation was carried out by determining the peroxide index value (PI) for 21 days. Four systems were prepared: soybean oil added extract in concentrations of 1000 and 3000 ppm, BHT in 1000 ppm and soybean oil free of antioxidant (control). The PI values oil jelly extract added at the concentrations were lower than the oil free of antioxidant during every 21 days, showing its ability to inhibit lipid oxidation, although in lower proportions of BHT. To the oil that was added the synthetic antioxidant BHT there was a delay of the formation of peroxides in $60 \%$, while the jelly extracts were equivalent, reducing around $43 \%$, in the concentrations tested. It is worth emphasizing the functional and health advantage of choosing to use the jelly extract as a natural antioxidant, even though it has a potential lower than the synthetic one.
\end{abstract}

Keywords: Antioxidants; Oxidative stability; Optimization.

\title{
Resumo
}

O objetivo do presente trabalho foi avaliar os efeitos dos extratos do fruto do jiló como um antioxidante natural em óleo de soja submetido ao teste de oxidação acelerada em estufa, bem como otimizar as melhores condições de extração dos compostos antioxidantes do jiló desidratado nas temperaturas de 40, 60, 80 e 100드. e assim avaliar a influência da temperatura na atividade antioxidante. A atividade antioxidante foi determinada pelo método de sequestro de radicais livres DPPH e os compostos fenólicos totais por meio do método Folin-Ciocalteu. As condições que forneceram o extrato do jiló com maior potencial antioxidante foram: desidratação à 60 으, utilizando $5,0 \mathrm{~g}$ da farinha, com $60 \mathrm{~mL}$ de metanol/água a $20 \%(\mathrm{v} / \mathrm{v})$ durante 24 horas e ao abrigo da luz. O monitoramento da oxidação do óleo foi realizado por meio da determinação do índice de peróxidos (IP) por 21 dias. Foram preparados quatro sistemas: óleo de soja adicionado de extrato nas concentrações de 1000 e 3000 ppm, BHT a 1000 ppm e o óleo de soja isento de antioxidantes (controle). Os valores de IP do óleo adicionado de extrato de jiló nas duas concentrações foram inferiores ao do óleo isento de antioxidantes durante todos os 21 dias, revelando sua capacidade de inibir a oxidação lipídica, embora em proporções inferiores a do BHT. Ao óleo que foi adicionado o antioxidante sintético BHT houve um retardo da formação de peróxidos em $60 \%$, enquanto os extratos de jiló foram equivalentes, reduzindo em torno de $43 \%$, nas concentrações testadas. Vale ressaltar a vantagem funcional e para a saúde de se optar pelo uso do extrato de jiló como antioxidante natural, mesmo possuindo potencial inferior ao sintético.

Palavras-chave: Antioxidantes; Estabilidade oxidativa; Otimização.

* Universidade Tecnológica Federal do Paraná, Coordenação do Curso de Licenciatura em Química (COLIQ), Rua Marcílio Dias, 635, Jardim Paraíso, CEP 86812-460, Apucarana-PR, Brasil.

$M$ liliandusman@utfpr.edu.br DOI: $\underline{10.21577 / 1984-6835.20170044}$

Rev. Virtual Quim. |Vol 9| | No. 2| |729-739| 


\section{Otimização do Processo de Extração de Compostos Fenólicos Antioxidantes do Jiló (Solanum gilo Radi) e Aplicação na Estabilidade Oxidativa do Óleo de Soja}

\section{Cristiana Fátima G. da Silva, Rúbia Michele Suzuki, Edmilson Antônio Canesin, Lilian Tatiani D. Tonin*}

Universidade Tecnológica Federal do Paraná, Coordenação do Curso de Licenciatura em Química (COLIQ), Rua Marcílio Dias, 635, Jardim Paraíso, CEP 86812-460, Apucarana-PR, Brasil.

* liliandusman@utfpr.edu.br

\section{Introdução}

\section{Material e Métodos}

2.1. Preparação das amostras

2.2. Compostos fenólicos totais

2.3. Atividade Antioxidante

2.4. Avaliação da estabilidade oxidativa

2.5. Índice de peróxido

2.6. Análise estatística

\section{Resultados e discussão}

4. Conclusões

\section{Introdução}

Os antioxidantes estão presentes ou são adicionados nas gorduras e alimentos com o objetivo de retardar a oxidação lipídica, mantendo intactas suas características sensoriais. ${ }^{1}$ A oxidação lipídica desenvolve sabores e odores desagradáveis nos alimentos, tornando-os impróprios para consumo. Este fenômeno também pode provocar outras alterações que afetam não só a qualidade nutricional, mas também a integridade e segurança dos alimentos. ${ }^{2}$

Os antioxidantes sintéticos butilhidroxianisol (BHA), butilhidroxitolueno (BHT), tri-hidroxi-butilfenona (THBP), propilgalato (PG) e terc-butilhidroquionona (TBHQ) (Figura 1), são empregados pela indústria alimentícia devido à sua alta eficiência na conservação de alimentos e baixo custo. ${ }^{2,3}$ Estes compostos, contudo, tem sido alvo de constante preocupação quanto à sua toxicidade, ocasionando busca por antioxidantes naturais que possam substituílos total ou parcialmente. 
<smiles>COc1ccc(O)c(C(C)(C)C)c1</smiles>

BHA<smiles>COc1ccc(O)cc1C(C)(C)C</smiles><smiles>CCCOC(=O)c1cc(O)c(O)c(O)c1</smiles><smiles>Cc1cc(C(C)(C)C)c(O)c(C(C)(C)C)c1</smiles>

$\mathrm{BHT}$<smiles>CCCC(=O)c1cc(O)c(O)cc1O</smiles>

TBHP

Figura 1. Estrutura dos antioxidantes sintéticos empregados pela indústria alimentícia

Antioxidantes naturais tendem a ser mais seguros, pois possuem propriedades antivirais, anti-inflamatórias, anticancerígenas, antimutagênicas, antitumoral, além de propriedades de proteção hepática. ${ }^{4}$ Numerosos antioxidantes naturais já foram isolados a partir de produtos naturais, tais como legumes, frutos, sementes, cereais e algas. ${ }^{5}$ Seu poder antioxidante muitas vezes é atribuído aos compostos fenólicos, essenciais no crescimento das plantas e eficientes na prevenção da autoxidação. ${ }^{6}$

O jiló (Solanum gilo Raddi) é uma hortaliça de porte herbáceo, pertencente à família Solanaceae. No Brasil, a introdução desta hortaliça ocorreu através dos escravos e atualmente o jiloeiro é bastante cultivado nos Estados do Rio de Janeiro, Espírito Santo,
São Paulo e Minas Gerais. ${ }^{7}$ Na literatura são citadas as sinonímias $S$. integrifoium $x$ aucts non Poir., S. paaschenianum $\mathrm{H}$. Winkl e também S. aethiopicum $\mathrm{L}^{8}$

O S. gilo é um fruto de consumo seletivo e pode ser utilizado na culinária. Em certas regiões brasileiras, é empregado na medicina popular na forma de infusão em aguardente com o intuito de combater gripes, resfriados e febre. Este fruto é recomendado ainda para dietas alimentares, por apresentar um baixo valor calórico, além de apresentar quantidades apreciáveis de sais minerais como cálcio, fósforo, ferro e vitaminas C e B5.

Nos estudos fitoquímicos de $S$. gilo foram isolados os compostos solasodina (1) e o 3-0$\beta$-glicosilsitosterol (2) (Figura 2), além de uma substância semelhante à acetilcolina no extrato aquoso do fruto do jiló. ${ }^{8-10}$<smiles>CC1CC1NC1CC1C1OC2CC3C4CC=C5CC(O)CC[C@]5(C)C4CCC3(C)C2[C@H]1C</smiles><smiles>CCC(CC[C@@H](C)C1CCC2[C@@H]3CC=C4C[C@@H](OC)CC[C@]4(C)C3CCC21C)C(C)C</smiles>

Figura 2. Compostos isolados de S. gilo 
Com o interesse na busca de antioxidantes naturais que venham substituir total ou parcialmente os sintéticos, o foco deste estudo objetivou determinar a atividade antioxidante do fruto do jiló e verificar a estabilidade oxidativa do óleo de soja adicionado do extrato mais ativo, em duas concentrações, por meio do processo de oxidação acelerada em estufa. O trabalho busca ainda otimizar as condições de extração dos compostos antioxidantes do jiló e avaliar a influência da temperatura de secagem neste parâmetro.

\section{Material e Métodos}

\subsection{Preparação das amostras}

Os frutos do jiló foram adquiridos em Novembro de 2012 num supermercado localizado na cidade de Apucarana - PR. Foram desidratados em estufa com circulação de ar nas temperaturas de $40^{\circ} \mathrm{C}$, $60^{\circ} \mathrm{C}, 80^{\circ} \mathrm{C}$ e $100^{\circ} \mathrm{C}$. Para isso foram inicialmente lavados, secos, fatiados em pequena espessura para facilitar a secagem e dispostos em bandejas. Após desidratação, os frutos in natura foram lavados, secos, triturados em liquidificador doméstico e armazenados sob-refrigeração para análises posteriores.

A otimização para a extração dos compostos antioxidantes foi realizada com as amostras desidratadas a $40^{\circ} \mathrm{C}$. Para a otimização foram utilizadas 5,0 g de amostra com volumes de 40,50,60, 70, 80 e $90 \mathrm{~mL}$ de metanol, durante 24 horas à temperatura ambiente e ao abrigo da luz. Os 6 extratos foram submetidos à análise de determinação de fenóis totais e atividade antioxidante. A segunda otimização utilizou $5,0 \mathrm{~g}$ de amostra com o volume de metanol que forneceu a melhor resposta, nos tempos de 2, 4, 6 e 24 horas, à temperatura ambiente e ao abrigo da luz. Os 4 extratos foram submetidos à análise de determinação de fenóis totais e atividade antioxidante. A última otimização determinou o tipo de solvente que extraísse a maior quantidade de compostos fenólicos antioxidantes, sendo utilizadas $5,0 \mathrm{~g}$ de amostra, com o volume dos solventes metanol, etanol, metanol/água $20 \%$ (v/v) e etanol/água $20 \%(\mathrm{v} / \mathrm{v})$ determinado na primeira otimização, à temperatura ambiente e ao abrigo da luz, num período de tempo determinado na segunda otimização. Os 4 extratos foram submetidos à análise de determinação de fenóis totais e atividade antioxidante.

\subsection{Compostos fenólicos totais}

A determinação do conteúdo de fenóis totais foi realizada de acordo com o método colorimétrico de Folin-Ciocalteu, segundo a metodologia descrita por Singleton e Rossi. ${ }^{11}$ As soluções dos extratos $(0,5 \mathrm{~mL})$ foram misturadas com 0,5 mL do reagente FolinCiocalteu, 1,0 mL de carbonato de sódio $20 \%$ $(\mathrm{m} / \mathrm{v})$ e $8,0 \mathrm{~mL}$ de água destilada. Para o branco não foi adicionado o reagente FolinCiocalteu, sendo este substituído por 0,5 $\mathrm{mL}$ de água destilada. A absorbância foi medida em espectrofotômetro VIS (DR2800 Hach) a $725 \mathrm{~nm}$ após vinte e cinco minutos de incubação ao abrigo da luz a temperatura ambiente e mais cinco minutos de centrifugação, em rotação de 10.000 rpm. O conteúdo de fenóis totais foi determinado por interpolação da absorbância das amostras frente a uma curva de calibração (y $\left.=3,0577 x+0,0445, R^{2}=0,9959\right)$ construída com o padrão ácido gálico e expresso em $\mathrm{mg}$ equivalente de ácido gálico por 100 gramas de amostra (mgEAG $100 \mathrm{~g}^{-1}$ ). Todas as determinações foram realizadas em triplicata.

\subsection{Atividade antioxidante}

A atividade antioxidante dos extratos do jiló foi realizada pelo método do radical DPPH (2,2-difenil-picrilhidrazila), descrito por Kulisicet al.. ${ }^{12} \mathrm{O}$ volume de $1,0 \mathrm{~mL}$ dos 
extratos, em diferentes concentrações, foi adicionado a 2,0 $\mathrm{mL}$ de uma solução de DPPH (40 $\mu \mathrm{g} \mathrm{mL}^{-1} ; 0,10 \mathrm{mmol} \mathrm{L}^{-1} \mathrm{em}$ metanol). Após 30 minutos de incubação, a absorbância foi determinada em espectrofotômetro VIS (DR 2800 - Hach) a $515 \mathrm{~nm}$. O aparelho foi zerado com metanol. O controle negativo foi preparado adicionando-se $1,0 \mathrm{~mL}$ de metanol a 2,0 $\mathrm{mL}$ da solução de DPPH. A porcentagem de inibição do radical DPPH foi calculada de acordo com a fórmula de Kulisic et al. ${ }^{12}$

$$
\% \text { Inibição }=\left[\left(A_{c}-A_{a}\right) / A_{c}\right] \times 100
$$

Onde:

$A_{c}$ corresponde a absorbância do controle negativo (solução DPPH + Metanol)

$A_{a}$ corresponde a absorbância da amostra após 30 minutos

A quantidade de amostra necessária para diminuir a absorbância do DPPH $\left(\mathrm{IC}_{50}\right)$ em $50 \%$ foi calculada graficamente. Foi plotado um gráfico de porcentagem de inibição (\%l) versus a concentração da amostra, e o valor de $I C_{50}$ foi obtido através da equação da reta.

\subsection{Avaliação da estabilidade oxidativa}

O óleo de soja sem adição de antioxidante foi adquirido da empresa COCAMAR da cidade de Maringá. Foram submetidos ao teste de oxidação acelerado em estufa a $65^{\circ} \mathrm{C}$, também conhecido como método de Schaal, quatro amostras: óleo de soja sem adição de antioxidantes, óleo de soja com adição de 1000 ppm de BHT e óleo de soja com adição de 1000 ppm e 3000 ppm de extrato metanol/água a $20 \%(\mathrm{v} / \mathrm{v})$ do jiló. Os tratamentos foram conduzidos por 25 dias em estufa aquecida, utilizando-se béqueres de $250 \mathrm{~mL}$ contendo $100 \mathrm{~mL}$ de amostra. As amostras foram tomadas nos intervalos de tempo de $0,3,7,9,11,14,16,18,21,23$ e 25 dias para determinação do índice de peróxidos.

\section{5. Índice de peróxido}

Durante a avaliação da estabilidade oxidativa foram coletadas, em triplicata, alíquotas para o acompanhamento do índice de peróxido, que foi determinado por titulação com tiossulfato de sódio pelo método AOAC Cd 8-53. ${ }^{13}$

Para a determinação do índice de peróxido, 2,5 gramas da amostra foram misturadas com $15 \mathrm{~mL}$ de ácido acético glacial/clorofórmio na proporção $3: 2(\mathrm{v} / \mathrm{v})$ e $0,5 \mathrm{~mL}$ de uma solução aquosa saturada de iodeto de potássio. Após agitação e 1 minuto de repouso, foram adicionados $15,0 \mathrm{~mL}$ de água destilada e $0,5 \mathrm{~mL}$ de uma solução aquosa de amido a $1 \%(\mathrm{~m} / \mathrm{v})$. As amostras foram tituladas com tiossulfato de sódio 0,01 mol $\mathrm{L}^{-1}$, e o volume de titulante gasto forneceu a concentração de peróxidos em meq $\mathrm{O}_{2} \mathrm{~kg}^{-1}$ através da fórmula:

$$
\mathrm{IP}=\frac{\mathrm{N} \text { da solução de tiossulfato de sódio } \times(\text { gasto na titulação }-\mathrm{V} \text { gasto na titulação do branco) }}{\text { Massa (gramas) }} \times 100
$$

\subsection{Análise estatística}

As análises estatísticas foram realizadas utilizando-se o software Statistica versão 8.0. ${ }^{14}$ Os resultados são apresentados como a média \pm desvio padrão, $n=3$. Foram considerados estatisticamente diferentes os resultados que apresentaram probabilidade de ocorrência da hipótese de nulidade menor que $5 \%(p \leq 0,05)$ aplicando-se Análise de Variância (ANOVA), seguido de comparações múltiplas pelo teste de Tukey. 


\section{Resultados e discussão}

O primeiro passo para otimizar a metodologia de extração de compostos fenólicos antioxidantes foi determinar a relação ideal entre o volume do solvente e a massa da amostra. Essa primeira etapa da otimização visa minimizar a geração de resíduos em laboratório, já que é um grave problema ambiental e deve ser reduzido ao mínimo sem perder a eficiência de extração. Portanto, nesta etapa experimental empregou-se o metanol, que é um solvente amplamente utilizado para extração de fitoquímicos. ${ }^{15}$

Alterando o volume de solvente metanol entre 40 e $90 \mathrm{~mL}$, foram observadas diferenças significativas $(p \leq 0,05)$ para o teor de compostos fenólicos totais, obtendo-se melhores resultados para o volume de $60 \mathrm{~mL}$ (Tabela 1). Já para a atividade antioxidante, os valores de $\mathrm{IC}_{50}$ não apresentaram diferenças significativas. Portanto, o volume de $60 \mathrm{~mL}$ extraiu o máximo de compostos fenólicos antioxidante com a mínima utilização de solvente possível.

Tabela 1. Conteúdo de fenóis totais e atividade antioxidante do jiló extraído com diferentes volumes de metanol

\begin{tabular}{ccc}
\hline Volume $(\mathbf{m L})$ & Fenóis Totais $\left(\mathbf{m g ~ E A G ~} \mathbf{~ 1 0 0 g}^{-1}\right)$ & $\mathbf{I C}_{50}\left(\mu \mathrm{g} \mathrm{mL}^{-1}\right)$ \\
\hline 40 & $303,2 \pm 1,97^{\mathrm{cd}}$ & $5165,5 \pm 40,5^{\mathrm{a}}$ \\
50 & $410,9 \pm 38,6^{\mathrm{b}}$ & $5138,3 \pm 231,2^{\mathrm{a}}$ \\
60 & $520,8 \pm 21,5^{\mathrm{a}}$ & $4786,2 \pm 57,6^{\mathrm{a}}$ \\
70 & $389,1 \pm 47,3^{\mathrm{bc}}$ & $4918,4 \pm 126,3^{\mathrm{a}}$ \\
80 & $344,5 \pm 21,2^{\text {bcd }}$ & $4673,0 \pm 372,6^{\mathrm{a}}$ \\
90 & $295,7 \pm 41,2^{\mathrm{d}}$ & $4754,4 \pm 85,6^{\mathrm{a}}$ \\
\hline
\end{tabular}

Valores representam a média \pm desvio padrão $(n=3)$. Médias seguidas de letras minúsculas diferentes na coluna diferem pelo teste de Tukey, significância $(p \leq 0,05)$.

Existe uma grande importância no estudo do período de maceração, que corresponde ao tempo que a amostra fica em contato com a solução extratora, pois influencia na melhor eficiência da extração. Neste trabalho, as amostras permaneceram em contato com 60 $\mathrm{mL}$ do solvente metanol nos tempos de 2,4 , 6 e 24 horas e o conteúdo de fenóis totais e atividade antioxidante foram determinados e seus valores estão apresentados na Tabela 2.

Tabela 2. Conteúdo de fenóis totais e atividade antioxidante do jiló extraído em períodos distintos de maceração com metanol

\begin{tabular}{ccc}
\hline Período (horas) & Fenóis Totais (mg EAG/100g) & IC $_{50}(\boldsymbol{\mu g} / \mathbf{m L})$ \\
\hline 2 & $201,1 \pm 31,2^{\mathrm{b}}$ & $10601,1 \pm 77,2^{\mathrm{a}}$ \\
4 & $340,2 \pm 8,65^{\mathrm{a}}$ & $10793,5 \pm 99,7^{\mathrm{a}}$ \\
6 & $364,7 \pm 3,41^{\mathrm{a}}$ & $9105,8 \pm 44,3^{\mathrm{b}}$ \\
24 & $372,8 \pm 38,6^{\mathrm{a}}$ & $7795,2 \pm 107,9^{\mathrm{c}}$ \\
\hline
\end{tabular}

Valores representam a média \pm desvio padrão $(n=3)$. Médias seguidas de letras minúsculas diferentes na coluna diferem pelo teste de Tukey, significância $(p \leq 0,05)$.

O tempo de 24 horas não apresentou diferença significativa $(p \leq 0,05)$ com os tempos de 4 e 6 horas para a determinação de compostos fenólicos totais, contudo a 
atividade antioxidante foi significativamente maior neste tempo $(p \leq 0,05)$, com um $\mathrm{IC}_{50}$ de 7795,16 $\mu \mathrm{gmL}^{-1}$. Nossos resultados demonstraram que quanto maior o tempo de interação amostra/solvente, maior a extração de compostos antioxidantes, sendo o tempo de 24 horas utilizado para preparação dos extratos do jiló.

Quatro sistemas de solventes foram avaliados para a extração de compostos fenólicos antioxidantes do jiló, empregando- se $60 \mathrm{~mL}$ de solvente e o tempo de 24 horas de maceração, condições ótimas encontradas neste trabalho. A atividade antioxidante e o teor de fenólicos totais variaram em função do solvente empregado (Tabela 3). Para ambas as análises o melhor resultado foi o sistema de solventes metanol/água a $20 \%$ (v/v), demonstrando uma maior eficiência de extração dos compostos fenólicos antioxidantes da matriz analisada.

Tabela 3. Conteúdo de fenóis totais e atividade antioxidante do jiló extraído em diferentes solventes

\begin{tabular}{ccc}
\hline Solvente & Fenóis Totais (mg EAG/100g) & IC $_{50}(\mu \mathrm{g} / \mathrm{mL})$ \\
\hline Metanol & $544,81 \pm 5,48^{\mathrm{c}}$ & $6064,1 \pm 150,9^{\mathrm{b}}$ \\
Metanol/Água a 20\% (v/v) & $830,6 \pm 16,2^{\mathrm{a}}$ & $4448,4 \pm 57,5^{\mathrm{c}}$ \\
Etanol & $208,7 \pm 11,1^{\mathrm{d}}$ & $59439,0 \pm 733,2^{\mathrm{a}}$ \\
Etanol/Água a 20\% (v/v) & $655,0 \pm 28,2^{\mathrm{b}}$ & $6777,2 \pm 32,0^{\mathrm{b}}$ \\
\hline
\end{tabular}

Valores representam a média \pm desvio padrão $(n=3)$. Médias seguidas de letras minúsculas diferentes na coluna diferem pelo teste de Tukey, significância $(p \leq 0,05)$.

Neste estudo não foi utilizada água pura para a extração de fenóis antioxidantes, pois poderia ser obtido um extrato com muitas impurezas (ácidos orgânicos, açúcares, proteínas solúveis), fato que geraria interferência na quantificação destes compostos. ${ }^{16} \mathrm{~A}$ água em combinação com outros solventes orgânicos contribui para criar um meio moderadamente polar, o que favorece a extração de polifenóis. ${ }^{17,18}$ Estes dados corroboram com Liu, Ang e Springer ${ }^{19}$ que sugerem que solventes com polaridades muito altas, muito baixas ou apolares não são bons extratores.

Não foram encontrados relatos na literatura a respeito do conteúdo de fenóis totais e atividade antioxidante da espécie $S$. gilo, apenas relatos para o gênero, como por exemplo Roesler et $a l^{20}$ estudaram o gênero Solanum lycocarpum (lobeira), Oliveira et al. ${ }^{21}$ analisaram o gênero Solanum tuberosum L. (batata inglesa) e Solanum melongina $\mathrm{L}$. (berinjela).
Os compostos fenólicos totais podem ser influenciados por fatores como: espécie estudada, práticas de cultivo, maturação, origem geográfica, estágio de crescimento, condições de colheita e processo de armazenamento. ${ }^{22}$ Esta sugestão está de acordo com os resultados encontrados neste trabalho, bem como, com os dados da literatura apresentados.

A fim de se verificar a influência da temperatura de secagem nos frutos da espécie $S$. gilo, amostras foram submetidas à desidratação nas temperaturas de $100^{\circ} \mathrm{C}$, $80^{\circ} \mathrm{C}$ e $60^{\circ} \mathrm{C}$. Os tempos de desidratação, assim como os resultados de fenóis totais e atividade antioxidante dos produtos desidratados e do jiló in natura estão expressos na Tabela 4. Foi possível observar uma significativa perda de massa, alcançando um rendimento de aproximadamente $11 \%$ para todas as temperaturas. Os tempos de secagem diminuíram significativamente com o aumento da temperatura. 
Tabela 4. Conteúdo de fenóis totais e atividade antioxidante dos extratos do jiló in natura e obtidos a partir da farinha desidratada a diferentes temperaturas

\begin{tabular}{|c|c|c|c|}
\hline Temperatura $\left({ }^{\circ} \mathrm{C}\right)$ & Tempo secagem (hs) & 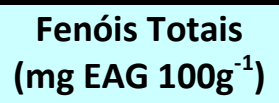 & $\mathrm{IC}_{50}\left(\mu \mathrm{g} \mathrm{mL}^{-1}\right)$ \\
\hline In natura & - & $77,01 \pm 0,78^{e}$ & $>80000$ \\
\hline 100 & 4,5 & $528,1 \pm 6,25^{d}$ & $12979,2 \pm 335,6^{a}$ \\
\hline 80 & 7,5 & $634,7 \pm 11,3^{c}$ & $6669,0 \pm 36,8^{b}$ \\
\hline 60 & 9,0 & $781,4 \pm 20,7^{b}$ & $4299,37 \pm 21,5^{d}$ \\
\hline 40 & 24 & $830,6 \pm 16,2^{a}$ & $4448,38 \pm 57,5^{c}$ \\
\hline
\end{tabular}

Valores representam a média \pm desvio padrão $(n=3)$. Médias seguidas de letras minúsculas diferentes na coluna diferem pelo teste de Tukey, significância $(p \leq 0,05)$.

O fruto in natura apresentou uma atividade antioxidante bastante reduzida (superior a $80000 \mu \mathrm{g} \mathrm{mL}^{-1}$ ) quando comparada com o fruto desidratado, demonstrando que a desidratação favoreceu a concentração dos componentes ativos do fruto.

Os valores mostraram que as temperaturas de secagem mais elevadas $\left(80^{\circ} \mathrm{C}\right.$ e $\left.100^{\circ} \mathrm{C}\right)$ apresentaram valores de $\mathrm{IC}_{50}$ significativamente maiores e valores de fenóis totais significativamente menores, quando comparados com as farinhas desidratadas à $40^{\circ} \mathrm{C}$ e $60^{\circ} \mathrm{C}$. Estes dados demonstram a perda do potencial antioxidante com o aumento da temperatura e a relação desta atividade com o conteúdo de fenóis totais.

De acordo com Tomaino et al., ${ }^{23}$ a secagem geralmente provoca a degradação dos antioxidantes que ocorrem naturalmente, devido à instabilidade destes compostos ao calor da secagem. A perda do conteúdo de compostos fenólicos com o aumento da temperatura de desidratação pode ser resultado da ligação destes com outros componentes do fruto do jiló, tais como proteínas, ou por causa da alteração da estrutura química com a secagem. ${ }^{24}$

A temperatura de $60^{\circ} \mathrm{C}$ apresentou menor conteúdo de fenóis totais, contudo, uma maior atividade antioxidante quando comparada com a secagem à $40^{\circ} \mathrm{C}$. Tal fato sugere que esta pode ser a temperatura mais adequada para desidratar o fruto do jiló.

A Tabela 5 apresenta os resultados de índice de peróxido (IP) do óleo de soja isento de antioxidante (controle), adicionado de antioxidante sintético BHT (1000 ppm), e adicionado do extrato otimizado do jiló $(5,00$ g de amostra com $60 \mathrm{~mL}$ de metanol/água a $20 \%(\mathrm{v} / \mathrm{v})$ durante 24 horas em repouso ao abrigo de luz, utilizando-se o jiló desidratada à $60^{\circ} \mathrm{C}$ ) nas concentrações de 1000 e 3000 ppm.

De acordo com os dados obtidos, o índice de peróxido aumentou em todos os tratamentos durante o período do teste, fato que é justificado pelo desenvolvimento de produtos primários da oxidação lipídica. Segundo a RDC N. ${ }^{\circ} 270 / 2005$ da ANVISA, a adequação de óleo de soja refinado para consumo é de, no máximo, 10 meq $\mathrm{O}_{2} \mathrm{Kg}^{-1}$ de índice de peróxido. ${ }^{25} \mathrm{~A}$ análise estatística revela que os tratamentos com os extratos de jiló nas duas concentrações foram mais eficientes que o BHT no terceiro dia. No sétimo dia, os extratos do jiló foram equivalentes ao BHT no controle da oxidação, retardando a formação de peróxidos e revelando boa eficácia na estabilização do óleo de soja.

A partir do 9o dia até o $21^{\circ}$ dia, observouse um efeito antioxidante do BHT superior 
aos dois tratamentos com jiló, exceto no $11^{\circ}$ dia em que o extrato na concentração de 3000 ppm foi equivalente ao BHT.

Iqbal e Bhanger ${ }^{26}$ relatam que a maioria dos antioxidantes inibe ou interrompe a deterioração oxidativa no seu estágio inicial, entretanto, são eficientes apenas por um período determinado de tempo. Isso se deve ao fato de que os antioxidantes se decompõem com o passar do tempo, resultando na perda de sua atividade protetora.

Os valores de índice de peróxidos do óleo adicionado de extrato de jiló nas duas concentrações foram inferiores ao do óleo isento de antioxidantes durante todos os 21 dias, revelando sua capacidade de inibir a oxidação lipídica, embora em proporções inferiores à inibição promovida pela presença do BHT.

Até o 14 dia de aquecimento o óleo de soja adicionado do extrato de jiló a 3000 ppm apresentou menor valor de índice de peróxidos que o óleo adicionado de extrato de jiló na concentração menor, demonstrando que o efeito protetor aumenta com a elevação de sua concentração. No 16 o dia não houve diferença significativa $(p<0,05)$ para IP nas duas concentrações do extrato e, nos últimos dias, ocorreu uma inversão de atividade, sendo que o óleo adicionado do extrato com menor concentração apresentou um maior efeito protetor.

O óleo adicionado do antioxidante sintético BHT retardou a formação de peróxidos em $60 \%$, enquanto os extratos de jiló reduziram em torno de 43\%, nas concentrações de 1000 e 3000 ppm, sendo esta proteção atribuída à ação antioxidante. Apesar de o BHT ter apresentado proteção superior ao óleo, nas concentrações estudadas, deve-se ressaltar a vantagem para a saúde e funcionalmente, de se optar pelo uso do extrato Metanol/Água a $20 \%$ (v/v) de jiló como antioxidante natural.

Tabela 5. Índice de peróxido (meq $\mathrm{O}_{2} \mathrm{~kg}^{-1}$ ) em óleo de soja adicionado de extrato de jiló e BHT, submetido ao teste da estufa a $65^{\circ} \mathrm{C}$

\begin{tabular}{ccccccccccc}
\hline Amostra & $\mathbf{C}$ (ppm) & $\mathbf{0}$ & $\mathbf{3}$ & $\mathbf{7}$ & $\mathbf{9}$ & $\mathbf{1 1}$ & $\mathbf{1 4}$ & $\mathbf{1 6}$ & $\mathbf{1 8}$ & $\mathbf{2 1}$ \\
\hline controle & - & $4,04^{\mathrm{g}, \mathrm{A}}$ & $6,04^{\mathrm{B}, \mathrm{C}}$ & $68,2^{\mathrm{f}, \mathrm{A}}$ & $102,4^{\mathrm{e}, \mathrm{A}}$ & $108,3^{\mathrm{e}, \mathrm{A}}$ & $133,4^{\mathrm{d}, \mathrm{A}}$ & $166,2^{\mathrm{C}, \mathrm{A}}$ & $230,5^{\mathrm{b}, \mathrm{A}}$ & $275,0^{\mathrm{a}, \mathrm{A}}$ \\
BHT & 1000 & $4,09^{\mathrm{f}, \mathrm{A}}$ & $8,91^{\mathrm{f}, \mathrm{A}}$ & $23,9^{\mathrm{e}, \mathrm{B}, \mathrm{C}}$ & $58,1^{\mathrm{d}, \mathrm{D}}$ & $44,5^{\mathrm{c}, \mathrm{d}, \mathrm{C}}$ & $67,8^{\mathrm{c}, \mathrm{D}}$ & $66,6^{\mathrm{c}, \mathrm{d}, \mathrm{C}}$ & $94,6^{\mathrm{b}, \mathrm{D}}$ & $109,3^{\mathrm{a}, \mathrm{C}}$ \\
& & & & & & & & & & \\
Jiló & 1000 & $4,18^{\mathrm{e}, \mathrm{A}}$ & $6,66^{\mathrm{e}, \mathrm{B}, \mathrm{C}}$ & $31,8^{\mathrm{d}, \mathrm{B}}$ & $73,2^{\mathrm{c}, \mathrm{B}}$ & $77,6^{\mathrm{c}, \mathrm{B}}$ & $107,5^{\mathrm{b}, \mathrm{B}}$ & $105,1^{\mathrm{b}, \mathrm{B}}$ & $107,5^{\mathrm{b}, \mathrm{C}}$ & $153,7^{\mathrm{b}, \mathrm{B}}$ \\
& & & & & & & & & & \\
Jiló & 3000 & $4,39^{\mathrm{g}, \mathrm{A}}$ & $7,17^{\mathrm{g}, \mathrm{B}}$ & $20,4^{\mathrm{f}, \mathrm{C}}$ & $66,9^{\mathrm{e}, \mathrm{C}}$ & $60,6^{\mathrm{e}, \mathrm{C}}$ & $92,2^{\mathrm{d}, \mathrm{C}}$ & $116,6^{\mathrm{c}, \mathrm{B}}$ & $126,3^{\mathrm{b}, \mathrm{B}}$ & $158,7^{\mathrm{a}, \mathrm{B}}$ \\
& & & & & & & & & &
\end{tabular}

a,b... (linha) - médias seguidas de mesma letra minúscula não diferem pelo teste de Tukey $(p<0,05)$.

A, B... (coluna) - médias seguidas de mesma letra maiúscula não diferem pelo teste de Tukey $(p<0,05)$.

\section{Conclusões}

A otimização da extração dos compostos fenólicos do fruto do jiló forneceu melhores valores de atividade antioxidante frente 0 DPPH nas seguintes condições: 5,00 $\mathrm{g}$ de amostra com $60 \mathrm{~mL}$ de metanol/água a $20 \%$ (v/v) durante 24 horas em repouso ao abrigo de luz, utilizando-se a farinha desidratada à $60 \circ \mathrm{C}$. 
Os extratos do jiló nas duas concentrações (1000 e 3000 ppm) mostraram-se eficientes na estabilidade oxidativa do óleo de soja nos primeiros 3 dias, com valores de índice de peróxido dentro da legislação brasileira. Observou-se ainda, em todos os ensaios de oxidação acelerada realizados, a dependência do desenvolvimento da oxidação lipídica em função do tempo de aquecimento.

O extrato de jiló, nas duas concentrações testadas, retardou a formação de peróxidos em torno de $43 \%$, mostrando-se eficiente, contudo com potencial inferior ao antioxidante sintético BHT, que inibiu em $60 \%$ a formação de peróxidos no óleo de soja testado. Vale ressaltar a vantagem funcional e para a saúde de se optar pelo uso do extrato Metanol/Água a $20 \%$ (v/v) de jiló como antioxidante natural, mesmo possuindo potencial inferior ao sintético. Estudos mais específicos envolvendo o extrato devem ser realizados para avaliar os componentes responsáveis pela ação antioxidante apresentada pelo mesmo.

\section{Referências Bibliográficas}

${ }^{1}$ Silva, A. C.; Oliveira, M. C.; Del Ré, P. V.; Jorge, N. Utilização de extrato de cogumelo como antioxidante natural em óleo vegetal. Ciência agrotecnologia 2009, 33, 1103. [CrossRef]

${ }^{2}$ Ramalho, V. C.; Jorge, N. Atividade antioxidante do $\alpha$-tocoferol e do extrato de alecrim em óleo de soja purificado. Revista Instituto Adolfo Lutz 2006, 65, 15. [Link]

${ }^{3}$ Pinho, O.; Ferreira, I. M. P. L. V. O.; Oliveira, M. B. P. P.; Ferreira, M. A. Quantification of synthetic phenolic antioxidants in liver patês. Food Chemistry 2000, 68, 353. [CrossRef]

${ }^{4}$ Lim, Y. Y.; Murtijaya, J. Antioxidant properties of Phyllanthus amarus extracts as affected by different drying methods. Food Science and Technology 2007, 40, 1664. [CrossRef]

${ }^{5}$ Pokorny, J. Natural antioxidants for food use. Trends in Food Science and Technology 1991, 2, 223. [CrossRef]
${ }^{6}$ Angelo, P. M.; Jorge, N. Compostos fenólicos em alimentos: uma breve revisão. Revista do Instituto Adolfo Lutz 2007, 66, 1. [Link]

${ }^{7}$ Pesagro-Rio; Emater-Rio. Recomendações para a cultura do jiló. Informe técnico, 18. Niterói, 1989. [Link]

${ }^{8}$ Vieira, R. F.; de Carvalho, L. D. A. F. Espécies do gênero Solanum produtoras de alcaloide sesteroidais. Revista Brasileira de Farmácia 1993, 74, 97. [Link]

${ }^{9}$ Almeida, E. R. Plantas medicinais brasileiras, São Paulo: Hemus, 1993. [Link]

${ }^{10}$ Silva, M. F.; Dissertação de Mestrado, Universidade Federal do Rio de Janeiro, 2004. [Link]

${ }^{11}$ Singleton, V. L.; Rossi, J. A. Colorimetry of total phenolic with phosphomolybdicphosphotungstic acid reagents. American Journal of Enology and Viticulture 1965, 16, 144. [Link]

${ }^{12}$ Kulisic, T.; Radonic, A.; Katalinic, V.; Milos, $M$. Use of different methods for testing antioxidative activity of oregano essential oil. Food Chemistry 2004, 85, 633. [CrossRef]

${ }^{13}$ ASSOCIATION OF OFFICIAL ANALYTICAL CHEMISTS (AOAC). Official methods of analysis of the association of official analytical chemists, 16a. ed., Arlington: AOAC, 1998. [Link]

${ }^{14}$ Statistica Software GraphPadInStat (2009). Versão 8.0. [Link]

${ }^{15}$ Vizzotto, M.; Pereira, M. C. Amora-preta (Rubus sp.): otimização do processo de extração para determinação de compostos fenólicos antioxidantes. Revista Brasileira de Fruticultura 2011, 33, 1209. [CrossRef]

${ }^{16}$ Chirinos, R.; Rogez, H.; Campos, D.; Pedreschi, R.; Larondelle, Y. Optimization of extraction conditions of antioxidant phenolic compounds from mashua (Tropaeolum tuberosum Ruiz \& Pavon) Tubers. Separation and Purification Technology 2007, 55, 217. [CrossRef]

${ }^{17}$ Lapornik, B.; Prosek, M.; Wondra, A. G. Comparison of extract prepared from plant by products using different solvents and extraction time. Journal of Food Engineering 2005, 71, 214. [CrossRef] 
${ }^{18}$ Liyana-Pathirana, C.; Shahidi, F. plums. Food Chemistry 2003, 81, 231. Optimization of extraction of phenolics [CrossRef]

compounds from wheat using response ${ }^{23}$ Tomaino, A.; Cimino, F.; Zimbalatti, V.; surfase methodology. Food Chemistry 2005, Venuti, V.; Sulfaro, V.; De Pasquale, A., Saija, 93, 45. [CrossRef]

${ }^{19}$ Liu, F. F.; Ang, C. Y.; Springer, D. Optimization of extraction condictions for active components in Hypericum perforatum using surface methodology. Journal of Agriculture and Food Chemistry 2000, 48, 3364. [CrossRef] [PubMed]

${ }^{20}$ Roesler, R.; Malta, L. G.; Carrasco, L. C.; Holanda, R. B.; Sousa, C. A. S.; Pastore, G. M. Atividade antioxidante de frutas do cerrado. Ciência e Tecnologia de Alimentos 2007, 27, 53. [CrossRef]

${ }^{21}$ Oliveira, M. S.; Dors, G. C.; Soares, L. A. S.; Badiale-Furlong, E. Atividade antioxidante e antifúngica de extratos vegetais. Alimentos $e$ Nutrição 2007, 18, 267. [Link]

${ }^{22} \mathrm{Kim}$, D. O.; Jeong, S. W.; Lee, C. Y. Antioxidant capacity of phenolic A. Influence of heating on antioxidant activity and the chemical composition of some spice essential oils. Food Chemistry 2005, 89, 549. [CrossRef]

${ }^{24} \mathrm{Qu}, \mathrm{W}$.; Pan, Z.; Ma, H. Extraction modeling and activities of antioxidants from pomegranate marc. Journal of Food Engineering 2010, 99, 16. [CrossRef]

${ }^{25}$ ANVISA - Agência Nacional de Vigilância Sanitária. Resolução RDC no 270, de 22 de setembro de 2005. Regulamento técnico para óleos vegetais, gorduras vegetais e cremes vegetais. Diário Oficial da União, Poder Executivo, de 23 de setembro de 2005. [Link] ${ }^{26}$ Iqbal, S.; Bhanger, M. I. Stabilization of sunflower oil by garlic extract during accelerated storage. Food Chemistry 2007, 100, 246. [CrossRef] 